\title{
Dual reciprocity boundary element method for iron corrosion in acidic solution
}

\author{
A. Peratta \\ Wessex Institute of Technology, Southampton, UK
}

\begin{abstract}
This paper presents a hybrid two dimensional Dual Reciprocity Boundary Element Method (DRBEM) for modelling the primary reactions of iron corrosion in an acidic solution. The main focus is on the establishment of the DRBEM approach for this kind of problems in which the mathematical formulation is based on the Planck-Nerst law, the conservation of charge and the conservation of mass for the species participating in the process. The model is capable to describe the transport process of $\mathrm{Fe}^{+2}$ and $\mathrm{H}^{+}$ions in the aqueous electrolyte driven by the instantaneous electric field that results from the existent charge distribution. The results of this work compare qualitatively well with previously established references.
\end{abstract}

\section{Introduction}

Numerical modelling of corrosion cells is necessary in order to understand the basic principles involved in the process. Even the simplest system with primary chemical reactions can represent a challenging problem from both the theoretical and numerical points of view. The high complexity of the phenomenon is mainly due to the non-linear coupling between the transport and electric field equations in the electrolyte, and more specifically in the region close to the anodic and cathodic nests. The understanding of the basic principles may lead to novel techniques for corrosion control, and may help to avoid expensive experimental setups.

The goal of this work is to establish a Dual Reciprocity Boundary Element Method (DRBEM) for solving iron corrosion problems in which the anodic and cathodic surfaces are well identified in advance. The conceptual model discussed in this work is sketched in Fig. 1.

In particular, this paper focuses in the primary reactions occurring in the anode and cathode, and defers the secondary reactions (i.e. rust formation) for 


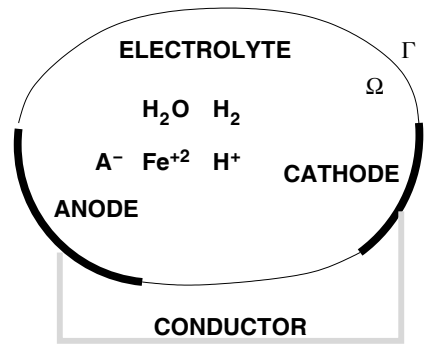

Figure 1: Conceptual model of the corrosion model to solve.

other models. The participating species are Iron ion $\left(\mathrm{Fe}^{+2}\right)$, Hydrogen ion $\left(\mathrm{H}^{+}\right)$ and a general ion $\mathrm{A}^{-}$coming from an additional dissolved acid. The problem consists in solving the time dependent transport equation for $\mathrm{Fe}^{+2}, \mathrm{H}^{+}$and $\mathrm{A}^{-}$, in the aqueous acidic electrolyte, coupled with the electric field equations due to the instant ionic distribution. The computational approach is the Dual Reciprocity Boundary Element Method (DRBEM). Numerical modelling with Boundary Element Methods (BEM) [1,2] is very attractive in the sense that it avoids domain discretisation and at the same time it uses the fundamental solution of the leading differential operator in the equation to solve. In addition, BEM applied to the time dependent advection-diffusion equation (A-DE) has been widely developed in the last decades and a large variety of efficient formulations were established [3,4]. Usually, the DRBEM yields a system of equations whose condition number grows with a certain power law of the number of degrees of freedom [5], thus imposing an upper practical limit to the size of the model to solve, this problem for DRBEM has not been completely sorted out so far, and the practical limitations of this formulation need to be explored in more detail.

\section{Governing equations}

The mathematical model is based on the work done by V. Botte et al [6], where a one-dimensional finite difference approach is employed to solve the iron corrosion problem in acidic aqueous solution. This section summarises the main theoretical aspects developed in the cited reference. By assuming low solute concentration and neglecting gravitational type convective motions in the electrolyte, the isothermal mass flux $\boldsymbol{J}_{k}$ of species $k$ in a non-dense aqueous electrolyte can be described by the Planck-Nernst law [7] according to:

$$
\boldsymbol{J}_{k}=-D_{k} \boldsymbol{\nabla} c_{k}-\frac{z_{k} F}{R_{0} T} c_{k} \boldsymbol{\nabla} \varphi
$$

where $D_{k}, c_{k}$ and $z_{k}$ are the diffusion coefficient, the concentration, and the charge number of $k$-th species in the aqueous solution, $F \sim 96500 \mathrm{sA} \mathrm{mol}^{-1}$ is the Faraday constant, $R_{0} \sim 8.314 \mathrm{JK}^{-1} \mathrm{~mol}^{-1}$ the universal gas constant, $T$ is the temperature of the system and $\varphi$ is the electric potential. 
The mass conservation equation can be expressed as:

$$
\frac{\partial c_{k}}{\partial t}+\nabla \cdot \boldsymbol{J}_{k}=\rho_{k}
$$

where $t$ is time and $\rho_{k}$ is a source term which represents the creation/annihilation of species $k$ due to secondary chemical reactions (i.e. rust production). The mass conservation equation leads to the time dependent A-DE with reaction term, see ref. [6], which describes the transport of species $k$ in the electrolyte:

$$
\frac{\partial c_{k}}{\partial t}+\nabla \cdot\left[-\left(\frac{z_{k} F D_{k}}{R_{0} T} \nabla \varphi\right) c_{k}\right]-D_{k} \nabla^{2} c_{k}=\rho_{k} .
$$

The instantaneous charge conservation equation which implies: $\boldsymbol{\nabla} \cdot\left(\sum z_{k} \boldsymbol{J}_{k}\right)=0$, leads to

$$
\boldsymbol{\nabla} \cdot\left[\left(\sum_{k} \frac{z_{k} F D_{k}}{R_{0} T} c_{k}\right) \nabla \varphi\right]=-\sum_{k} D_{k} \nabla^{2} c_{k}
$$

The boundary conditions for the electric problem are imposed by means of the dominant anode and cathode reactions: $\mathrm{Fe} \rightarrow \mathrm{Fe}^{+2}+2 e^{-}$and $2 \mathrm{H}^{+}+2 e^{-} \rightarrow \mathrm{H}_{2}$, respectively. Then the normal current in the anode can be modelled by means of a simplified Butler-Volmer equation with transfer coefficient equal to $1 / 2$ :

$$
i_{A}=2 i_{0} \sinh \left[\frac{z_{F e^{+2}} F}{R_{0} T} \eta_{A}\right]
$$

where $i_{A}$ is the current density of the anode (used as boundary condition for the electrical problem), $i_{0}$ is obtained experimentally, and

$$
\eta_{A}=\delta \varphi_{A}-E_{A}
$$

is the anode over-voltage, where $\delta \varphi$ is the difference of electric potential between the anode and the adjacent electrolyte film, and $E_{A}$ is the electrode potential at zero current, computed as:

$$
E_{A}=E_{0}+\frac{R_{0} T}{z_{F e^{+2}}} \ln \left[c_{F e^{+2}}\right]
$$

Once the current density at the anode is found in terms of the over-voltage and iron ion concentration, the mass flux given by eq. (2) can be obtained, thus yielding the necessary boundary conditions for the transport problem.

When an acid is added to the solution, it dissociates according to $H A \rightarrow$ $\mathrm{H}^{+}+\mathrm{A}^{-}$, thus reducing the $\mathrm{pH}$ in the electrolyte [6]. Then, $c_{A^{-}}=c_{F e^{+2}}+c_{H^{+}}$. 


\section{Dual Reciprocity Boundary Element Method}

The governing equations for the electric potential and the solute transport for each species can all be casted into Poisson-like equations of the form:

$$
\nabla^{2} c_{k}(\mathbf{x}, \mathbf{t})=b(\mathbf{x}, t) \quad \mathbf{x} \in \Omega,
$$

defined in the integration domain $\Omega$ with boundary $\Gamma=\partial \Omega$, where the right hand side (RHS) term $b(\boldsymbol{x}, t)$ can be regarded as a generic source term to be defined later for each problem. Then, the DRBEM [3, 8] is applied in order to convert this equation into an algebraic system of linear equations which require boundary only discretisation. Following the well established boundary integral approach, the weighted boundary integral expression of eq. (8) with function $G$ yields [2]:

$$
\begin{aligned}
\mu_{i} c_{k}\left(\boldsymbol{x}_{i}\right)+\int_{\Gamma} & \left(\frac{\partial G\left(\boldsymbol{x}_{i}, \boldsymbol{x}\right)}{\partial \hat{n}} c_{k}(\boldsymbol{x})-G\left(\boldsymbol{x}_{i}, \boldsymbol{x}\right) \frac{\partial c_{k}(\boldsymbol{x})}{\partial \hat{n}}\right) d \Gamma= \\
& \int_{\Omega} G\left(\boldsymbol{x}_{i}, \boldsymbol{x}\right) b(\boldsymbol{x}, t) d \Omega
\end{aligned}
$$

where $\mu_{i}=1 / 2$ for smooth boundaries, and the weight function $G$ is the fundamental solution of Laplace equation in 2D: $\nabla^{2} G\left(\boldsymbol{x}_{i}, \boldsymbol{x}_{j}\right)+\delta\left(x_{i}, x_{j}\right)=0$. The domain integral appearing in the RHS of eq. (9) is treated with the Dual Reciprocity Method (DRM) [8]. In this work we have employed a set of $N_{r}$ radial basis functions (RBF) $f_{i}\left(\boldsymbol{x}_{j}\right)=\left\{1, r_{i j}^{2} \ln \left(r_{i j}\right), x_{j}, y_{j}\right\}$ known as Augmented Thin Plate Splines (ATPS) in order to approximate $b$ as:

$$
b(\boldsymbol{x}, t) \approx \sum_{i=1}^{N_{r}} a_{i}(t) f_{i}(\boldsymbol{x}),
$$

where $r_{i j}$ is the distance between the source and field points denoted by indices $i$ and $j$, respectively, and $a_{j}(t)$ are the interpolating coefficients to be adjusted in order to minimise the error between $b$ and its approximation. The DRM is based on the fact that the RBFs can be considered as source terms of potential functions $\hat{u}$ defined by: $\nabla^{2} \hat{u}_{j}\left(x_{i}\right)=f_{i j}$. Henceforth, the RHS of eq. (9) can be integrated by parts in order to obtain a boundary-only integral equation of the form:

$$
\mu_{i} c_{k}+\int_{\Gamma}\left(\frac{\partial G}{\partial \hat{n}} c_{k}-G \frac{\partial c_{k}}{\partial \hat{n}}\right) d \Gamma=\sum_{j=1}^{N_{r}} a_{j}\left[\mu_{\xi} \hat{u}+\int_{\Gamma}\left(\frac{\partial G}{\partial \hat{n}} \hat{u}-G \frac{\partial \hat{u}}{\partial \hat{n}}\right) d \Gamma\right]
$$

Details of this approach can be found in refs. [5, 8]. In the standard BEM matrix notation, eq. (11) can be expressed as:

$$
\left.\left(\mathbf{H}\left\{\boldsymbol{c}_{\boldsymbol{k}}\right\}-\mathbf{G} \partial_{\mathbf{n}}\left\{\boldsymbol{c}_{\boldsymbol{k}}\right\}\right)=(\mathbf{H} \hat{\boldsymbol{U}}-\mathbf{G} \hat{\mathbf{Q}}\}\right)\{\boldsymbol{a}\},
$$

where $\boldsymbol{H}, \boldsymbol{G}, \hat{\boldsymbol{U}}$ and $\hat{\boldsymbol{Q}}$ are the usual DRBEM collocation matrices [9, 8, 2], $\left\{\boldsymbol{c}_{\boldsymbol{k}}\right\}=c_{k}\left(\boldsymbol{x}_{i}, t\right)$ is a 1-column array of $c_{k}$ evaluated at the collocation nodes, and 
the coefficients $\{\boldsymbol{a}\}=a_{j}$ can be determined inverting the linear eq. (10) provided that $\left\{\boldsymbol{b}_{\boldsymbol{i}}\right\}$ is known at the collocation nodes:

$$
\{\boldsymbol{a}\}=\boldsymbol{F}^{-1}\{\boldsymbol{b}\} .
$$

Then, eq. (12) becomes: $\mathbf{H} \mathbf{c}_{\mathbf{k}}-\mathbf{G} \partial_{\mathbf{n}} \mathbf{c}_{\mathbf{k}}=\mathbf{S} \boldsymbol{b}$, where $\mathbf{S}:=(\mathbf{H} \hat{\mathbf{U}}-\mathbf{G} \hat{\mathbf{Q}}) \mathbf{F}^{-1}$, $\mathbf{S} \in R^{M \times M}$, and $\mathbf{F}=f_{i j}, \in R^{(M+3) \times(M+3)}$ is the 2D ATPS RBFF matrix. Then, $\hat{\mathbf{U}}=\hat{u}_{i j}, \in R^{M \times(M+3)}$ and $\hat{\mathbf{Q}}=\partial \hat{u}_{i j} / \partial \hat{n}_{i} \in R^{N \times(M+3)}$ are the usual DRM matrices. $N$ is the number of freedom nodes used to discretise $\Gamma, M=N+L$, and $L$ is the number of DRM nodes in $\Omega$. Next, we express the 1-column array $\{\boldsymbol{b}\}$ representing the RHS term evaluated at the collocation nodes in a way that results suitable for the DRM treatment. The time dependent term is approximated as:

$$
\frac{\partial c_{k}}{\partial t} \approx \frac{c_{k}^{m+1}-c_{k}^{m}}{\delta t} ; \quad c_{k}^{m}=\theta c_{k}(t+\delta t)+(1-\theta) c_{k}(t)
$$

where index $m$ represents the time level and $\theta$ can be adjusted between 0 and 1 in order to tune the time integration scheme between fully explicit $(\theta=0)$ and implicit $(\theta=1)$. The convective term is split into the following two terms: $\boldsymbol{\nabla} \cdot\left(\boldsymbol{V} c_{k}\right)=(\boldsymbol{V} \cdot \boldsymbol{\nabla}) c_{k}+(\boldsymbol{\nabla} \cdot \boldsymbol{V}) c_{k}$. The first one on the RHS represents advection in incompressible media, i.e. $\boldsymbol{\nabla} \cdot \boldsymbol{V}=0$, while the second one can be regarded as a first order reaction term, provided that $\nabla \cdot V$ is known in advance.

\section{Computational implementation}

This section outlines the computational implementation of the simple iron corrosion process in a two dimensional cell. The problem is solved with an iterative two-stage sequential approach. The time integration scheme involves a finite difference approach with two time levels. First, the electric field is computed with the most updated values of species concentration, second the transport problem for each species is solved. Then, a new time level can be solved once the electric and transport problems are consistent with each other at present time level.

The integration domain is discretised with a mixed unstructured mesh, where some regions may be decomposed into many sub-domains and some others may be discretised only in their boundary. The former is known as multi-domain region while the latter is identified with single domain region. The assembly of multi and single-domain regions into the same problem provides a suitable pre-processing flexibility which allows the treatment of complicated geometries. In this work, the characteristic size of a cell in the multi-domain approach is $\sim 0.01 \mathrm{~m}$.

\subsection{Electric problem}

The electric problem at time level $m$ can be expressed as:

$$
\nabla^{2} \varphi^{m}=b_{\text {elec }} .
$$


The source term is expressed in terms of the concentrations found in the last time level according to:

$$
b_{\mathrm{elec}}=-\frac{\sum_{k} D_{k} \nabla^{2} c_{k}^{m-1}}{\sum_{k} \alpha_{k} c_{k}^{m-1}} .
$$

For simplicity, the DRBEM is implemented throughout a multi-domain approach in which instant diffusion coefficients can be regarded as piecewise constant isotropic and homogeneous in each sub-domain. After applying collocation DRBEM, the discrete version of the electric problem becomes:

$$
\boldsymbol{H}\{\boldsymbol{\varphi}\}+\boldsymbol{G}\{\boldsymbol{q}\}=\boldsymbol{S}\left\{\boldsymbol{b}_{\text {elec }}\right\} .
$$

The denominator in eq. (16) represents an apparently instant diffusion coefficient $D_{e}$ for the electric problem, and $\boldsymbol{q}=-D_{e} \boldsymbol{\nabla} \varphi \cdot \hat{\boldsymbol{n}}$ can be regarded as a current density, to be conserved throughout the interface between two sub-domains.

\subsection{Transport problem}

The second stage solves the transport problem for each chemical species involved. The transport problem can be formulated by casting the transport equation eq. (3) into a Poisson-like equation as follows:

$$
\nabla^{2} c_{k}(\mathbf{x}, \mathbf{t})=b_{\text {tran }}
$$

where the source term $b_{\text {tran }}$ given by:

$$
b_{\text {tran }}=\frac{1}{D_{k}}\left[\frac{\partial c_{k}}{\partial t}-\nabla \cdot\left(\boldsymbol{V} c_{k}\right)-\rho_{k}\right] \text {, }
$$

and the apparent convective velocity is defined as: $\boldsymbol{V}=\frac{z_{k} F D_{k}}{R_{0} T} \boldsymbol{E}$, where $\boldsymbol{E}=\boldsymbol{\nabla} \varphi$ is the electric field in the electrolyte. After applying the collocation DRBEM technique, the integral matrix equation per sub-domain becomes:

$$
\mathbf{H}\left\{\mathbf{c}_{\mathbf{k}}\right\}-\mathbf{G}\left\{\partial_{\mathbf{n}} \mathbf{c}_{\mathbf{k}}\right\}=\frac{\mathbf{S}}{D_{f}}\left[\frac{\partial}{\partial t}+\sum_{p=1}^{2}\left(\mathbf{V}_{p} \cdot \mathbf{T}_{p}\right)+\nabla \cdot \boldsymbol{V}\right]\left\{\mathbf{c}_{\mathbf{k}}\right\},
$$

where the following matrices were employed [5, 9]: $\mathbf{V}_{p}=\operatorname{diag}\left\{v_{p}\right\}$ and $\mathbf{T}_{p}=$ $\nabla_{p} \mathbf{F} \cdot \mathbf{F}^{-1}$; where $\mathbf{V} \in R^{M \times M \times 3}$ and $\mathbf{T} \in R^{M \times M \times 3}$, see ref. [9] for details. By applying same procedure as in [9] a close linear system of equations of the form

$$
\boldsymbol{A}_{\mathrm{TRAN}} \boldsymbol{x}=\boldsymbol{B}
$$

can be obtained, in which the solution represents fluxes and potentials at the boundary of the integration domain. 


\subsection{Coupling strategy}

The coupling strategy in each time level can then be summarised as follows:

1. Define initial conditions for $c_{k}$ obtained from the previous time level

2. Compute $b_{\text {elec }}$ with the most updated $c_{k}$ field

3. Update boundary conditions with Butler-Volmer equation (5)

4. Solve electric problem given by eq. (15)

5. Use the electric field in order to compute effective velocity $\boldsymbol{V}$

6. Compute $b_{\text {tran }}$ with the most updated $\varphi$ and $\boldsymbol{E}$

7. Solve A-DE system given by eq. (21) for each species

8. If $c_{k}$ is consistent with $\varphi$ stop, otherwise repeat step 2

The consistency is checked if the relative error of $c_{k}$ between two subsequent iterations $L$ and $L-1$ is smaller than a small arbitrary factor, i.e. $\sum\left|c_{k}^{L}-c_{k}^{(L-1)}\right|<$ $\varepsilon c_{k}^{L}$, and $\varepsilon \sim 0.1$.

\section{Results}

This section presents the numerical results obtained for two cases: in the first one, the cathode and anode are separated by a homogeneous column of electrolyte. In this example, symmetrical lateral boundary conditions, have been imposed in order to retrieve one-dimensional results. This is done in order to compare results with the work of Botte et al. [6].

The second example, has non-symmetrical conditions and describes qualitatively the behaviour of a 2D corrosion cell with two adjacent electrodes.

\subsection{One-dimensional example}

This example consists of a rectangular domain of $0.1 \mathrm{~m} \times 0.025 \mathrm{~m}$, with its larger dimension oriented in $x$ direction. The anode is placed on the left boundary $(x=0)$ while the cathode is on the right $(x=0.1 m)$ as shown in Figure 2 .

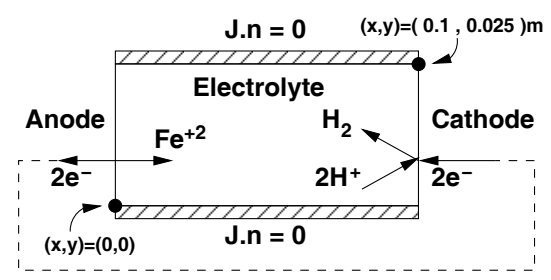

Figure 2: Conceptual model of the corrosion cell in example 1.

Impermeable conditions, i.e. $\boldsymbol{J} \cdot \hat{\boldsymbol{n}}=0$, were imposed on both top and bottom boundaries. The simulation parameters are summarised in table 1. Figure 3 shows the concentration profiles of $\mathrm{Fe}^{+2}$ and $\mathrm{H}^{+}$ions in the electrolyte at different time levels. The concentration of $\mathrm{Fe}^{+2}$ near the anode increases with time and 
Table 1: Simulation parameters.

\begin{tabular}{llll}
\hline Description & Symbol & \multicolumn{2}{c}{ Value } \\
\hline Concentration of $F e^{+2}$ at $t=0$ & $c_{F e^{+2}}(x, t=0)$ & $1 \times 10^{-14}$ & $\mathrm{~mol} / \mathrm{m}^{3}$ \\
Concentration of $H^{+}$at $t=0$ & $c_{H^{+}}(x, t=0)$ & $1 \times 10^{-3}$ & $\mathrm{~mol} / \mathrm{m}^{3}$ \\
Time step & $\delta t$ & $2 \times 10^{1}$ & $\mathrm{~s}$ \\
Exchange anode current density & $i_{0}$ & $1 \times 10^{1}$ & $\mathrm{~A} / \mathrm{m}^{2}$ \\
Anode reaction potential & $E_{0}$ & 1.44 & $\mathrm{~V}$ \\
Diffusion coefficient $F e^{+2}$ & $D_{F e^{+2}}$ & $5 \times 10^{-6}$ & $\mathrm{~m}^{2} / \mathrm{s}$ \\
Diffusion coefficient $H^{+}$ & $D_{H^{+}}$ & $1 \times 10^{-6}$ & $\mathrm{~m}^{2} / \mathrm{s}$ \\
Temperature & $T$ & 300 & $\mathrm{~K}$ \\
\hline
\end{tabular}

propagates towards the bulk of the solution as time passes by. On the other hand, the concentration of $\mathrm{H}^{+}$decreases with time and the maximum of its distribution moves towards the cathode at a very slow rate, where it is consumed in the cathode. The slow motion of the distribution of $\mathrm{H}^{+}$towards the cathode seems to be driven by the instant electric field. Figure 4 shows the concentration profiles of species $A^{-}$at different time levels. Figure 4 (right) shows the electric potential profile along the electrolyte at different times $3 h, 6 h, 9 h 12 h$ and $15 h$. it can be observed that the solution in the bulk of the electrolyte is quite close to the solution of the Laplacian equation, i.e. the gradient of concentration does not play a significant role in the electric problem far from the electrodes.

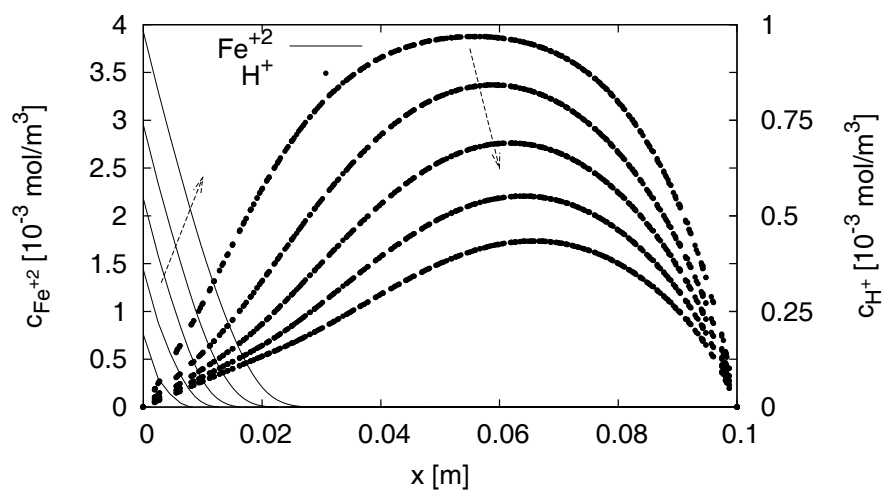

Figure 3: Concentration profiles of $\mathrm{Fe}^{+2}\left(c_{\mathrm{Fe}} e^{+2}-\right.$ left axis $)$ and $\mathrm{H}^{+}\left(c_{H^{+}}\right)$in $10^{-3} \mathrm{~mol} / \mathrm{m}^{3}$ at times $3 h, 6 h, 9 h 12 h$ and $15 h$. The arrows indicate the time direction. 

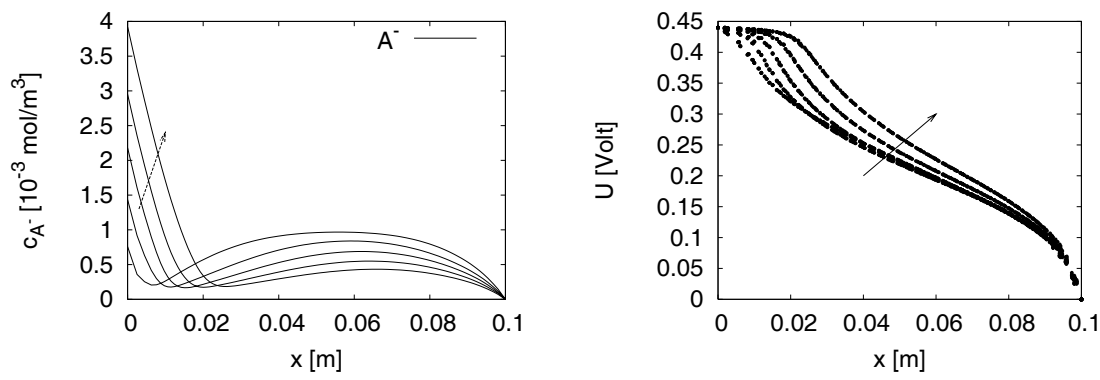

Figure 4: Concentration profiles of $A^{-}$ion $\left(c_{A^{-}}\right)$(left), and electric potential profiles (right) at times $3 h, 6 h, 9 h 12 h$ and $15 h$. The arrows indicate positive time direction.

\subsection{Two dimensional example}

The integration domain in this example is shown in Figure 5(a). It consists of a sample of $10 \mathrm{~cm} \times 5 \mathrm{~cm}$. The initial conditions were assumed in the same way as in the previous example. The boundary conditions for the anode were given according to the Butler-Volmer equation eq. (5) and the over-voltage was updated at each time step. The cathodic current density was the same as the anodic, in view of the global charge conservation equation. This can be done because all other boundary conditions apart from the anodic and cathodic surfaces have assigned zero normal current. Fig. 5(a) shows the concentration distribution of $\mathrm{Fe}^{+2}$ at time $t=1 \mathrm{~h}$. Fig. 5(b) shows the corresponding electric potential, the numbers associated with the iso-lines represent concentration in $\left[\mathrm{mol} / \mathrm{m}^{3}\right]$. The numbers inside the figure indicate potential in [V].

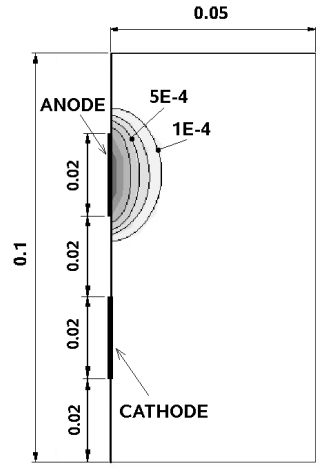

(a)

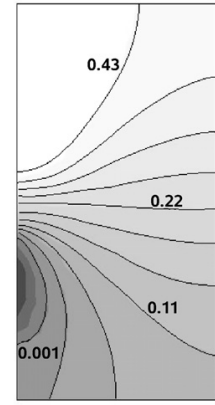

(b)

Figure 5: (a) Concentration of $\mathrm{Fe}^{+2}$, and (b) electric potential at time $t=1 \mathrm{~h}$. 


\section{Conclusions}

An iterative approach based on the multi-domain DRBEM for solving an iron corrosion model in acidic aquous electrolyte has been established and successfully implemented for two-dimensional problems.

The results obtained compare qualitatively well with previous publications [6], although more study is required in order to represent properly the thin layer of electrolyte close to the electrodes.

Further extensions of this work will involve the solution of the secondary reactions that yield rust and the study of the influence of external electric fields. This is in order to assess the impact of environmental electric pollution on simple corrosion cells.

\section{References}

[1] C.A. Brebbia and J. Dominguez. Boundary Elements, An Introductory Course. Second Edition. Computational Mechanics Publications, McGraw-Hill, New York Colorado San Francisco Springs Mexico Montreal Oklahoma City San Juan Toronto, 1992.

[2] C.A. Brebbia, J. C. Telles, and L. C. Wrobel. Boundary Elements Techniques. Springer-Verlag, Berlin, Heidelberg New York and Tokio, 1984.

[3] L.C. Wrobel and D. B.De Figueiredo. A dual reciprocity boundary element formulation for convection-diffusion problems with variable velocity fields. Engng Analysis with Boundary Elements, 8(6):312-319, 1991.

[4] Z. H. Qiu, L. C. Wrobel, and H. Power. An evaluation of boundary element schemes for convection-diffusion problems. In C. A. Brebbia and J.J. Rencis, editors, Boundary Elements XV, Transactions on Modelling and Simulation, volume 1. Wessex Institute of Technology, UK and Worcester Polytechnic Institute, USA, WIT Press, 1993.

[5] A. Peratta. BEM applied to Flow and Transport in Fractured Porous Media. PhD thesis, University of Wales - Wessex Institute of Technology, Southampton, UK, December 2004.

[6] V. Botte, D. Mansutti, and A.Pascarelli. Numerical modelling of iron corrosion due to an acidic aqueous solution. Applied Numerical Mathematics, 55:253263, June 2005.

[7] S. Furini, F. Zerbetto, and S. Cavalcanti. A numerical solver of 3D poisson nernst planck equations for functional studies of ion channels. In M. Ursino, editor, Modelling in Medicine and Biology, volume 2 of Advances in Bioengineering, Vol 2, pages 111-120, University of Bologna, Italy, 2005. WIT Press.

[8] P.W. Partridge, C.A. Brebbia, and L.C. Wrobel. The Dual Recoprocity Boundary Elements Method. Computational Mechanics Publications, Southampton Boston, 1992.

[9] A. Peratta and V. Popov. A new scheme for numerical modelling of flow and transport processes in $3 \mathrm{~d}$ fractured porous media. Advances in Water Resources, 29:42-61, 2006. 